\title{
Membranes, Electrodes, and Membrane-Electrodes Assemblies Analyzed before and after Operation by Atomic Force Microscopy
}

\author{
Tobias Morawietz ${ }^{\mathrm{a}}$, Michael Handl ${ }^{\mathrm{a}}$, Matthias Simolka ${ }^{\mathrm{a}}$, K. Andreas Friedrich ${ }^{\mathrm{b}}$, \\ Renate Hiesgen $^{\text {a }}$ *
}

\author{
${ }^{a}$ University of Applied Sciences Esslingen, Kanalstrasse 33, 73728 Esslingen, Germany \\ ${ }^{\mathrm{b}}$ German Aerospace Center, Institute of Engineering Thermodynamics, \\ Pfaffenwaldring 38-40, 70569 Stuttgart, Germany \\ *renate.hiesgen@hs-esslingen.de
}

\begin{abstract}
Material-sensitive and conductive atomic force microscopy was applied to the investigation of cross sections of membraneelectrode-assemblies (MEA) at operating conditions, $80 \%$ relative humidity and $75^{\circ} \mathrm{C}$, before and after operation. The ionomer content inside the electrodes could be measured due to their characteristic mechanical, chemical and physical properties. By surface potential measurements across MEAs after degrading fuel cell operation, a severe influence of the re-deposited platinum on the potential distribution was found.
\end{abstract}

Fuel cells are an attractive converter of chemical to electrical energy for automotive application because of their high theoretical efficiency and the easy use of renewable hydrogen gas as energy source. For such an application, a long enough operation durability is needed in the order of at least $4000 \mathrm{~h}$. There are still fundamental questions of stability of the components. The main component, the membrane-electrode-assembly (MEA) consists of an ionically conductive solid state ionomer sandwiched by two electrodes at the sides. The electrodes, anode and cathode, where the two electrochemical reactions take place, are complex composites fulfilling many functions. Typically, they consist of catalyst-covered mesoporous carbon, ionomer as binder, and pores. Mostly platinum or platinum alloys are used as catalyst with a size of a few nanometers. The carbon particles have a size of approximately $30-50 \mathrm{~nm}$. The reactions take place at the catalyst particles and the carbon provides electronic conductivity through the electrode. For the conduction of the ions, protons in acid ionomers, the ionomer plays a crucial role as ionic conductor and binder in the electrode. In contrast, a thick ionomer layer provides a large resistance to gas diffusion from the pore to the catalyst surface. Due to the sluggish oxygen reduction reaction, also oxygen transport resistances are dominant and the low solubility of oxygen inside the ionomer is the main problem. Because of their structural complexity considerable uncertainty exists about phase distribution inside the electrodes. The ionomer distribution has been mainly investigated with electron microcopy techniques. While the influence of beam damage could be significantly reduced with new instruments (1), high-resolution investigations by electron microscopies require vacuum conditions. The major drawback and source of artefacts is 
the significant shrinkage of the water-containing ionomers under these conditions. The distribution and homogeneity of thin films of Nafion have been frequently investigated with SEM/TEM (compare the review article Holdcroft and citation therein (2)). Todays knowledge retrieved from transmission electron microscopy (TEM) is an estimated mean thickness of Nafion layers surrounding carbon particles of 5-10 $\mathrm{nm}$ (3)

In a recent publication using a 3-D tomographic electron microscopy technique (HAADF), a mean thickness of $7 \mathrm{~nm}$ was reported as the layer thickness around carbon particles, also measured in vaccuum at a processed sample, scratched off from the electrode (4).

Using material-sensitive and conductive atomic force microcopy, samples can be investigated under controlled high humidity and at elevated temperatures, close to operation conditions.In this study, were report on the investigtion of cross sections of MEAs and electrodes in humid environment. In addition to the topography data, the material-sensitive AFM imaging delivers the mapping of adhesion forces, stiffness, and deformation of the surface (5). The ionomer can be distinguished by it large difference in adhesion force and stiffness since the contrast difference is quite high compared with carbon or platium (lowest). The investigation can be performed on cross-sections of real electrodes without further processing. With AFM, we can show images of the internal of the MEA at near operating conditions of a technical fuel cell.

\section{Experimental}

All images were measured using a Bruker multimode 8 AFM, equipped with PeakForce $\mathrm{QNM}{ }^{\circledR}$ and PeakForce TUNA ${ }^{\circledR}$ tapping mode. The images were performed on cross sections of MEA before and after operation prepared by microtome. The MEAs were composed of a reinforced ionomer membrane, either Nafion or Aquivion as ionomer. The same ionomer was present in the electrodes. The Pt loading was 0.6 $\mathrm{mg}_{\mathrm{Pt}} / \mathrm{cm}^{2}$. Cutted MEA pieces were embedded into Teromix (BASF, Ludwigshafen, Germany) for stabilization of the compound and the sandwich was microtomed after curing. The Teromix did not infiltrate significantly the sample due to its high viscosity but was effective as support of the sample. For one sample, two MEAs were embedded together for direct comparison of anode and cathode. The measurements were performed at controlled humidity and temperature, indicated in the figure cation. For the investigation of an aged MEA, it was operated for $200 \mathrm{~h}$ at $80^{\circ} \mathrm{C}$ and $90 \% \mathrm{RH}$ (stoich. $1.5 / 2$ ) in $\mathrm{H}_{2}$ /air. For current measurements, a Pt catalyst containing back-contact was provided and a Pt-coated AFM probe was used. For recording of an electronic current, a voltage of approximately $0.05 \mathrm{~V}$ was applied. Ionic current measurements were performed at humid conditions with an applied voltage of approximately $1.5 \mathrm{~V}$, with respect to the back contact. The current in tapping mode was averaged by the built-in lock-in amplifier. In the PeakForce QNM ${ }^{\circledR}$ mode additional to the height information, mechanical properties as adhesion force, stiffness (DMT modulus), and deformation are evaluated from the force-separation curve, recorded during approach and retraction of the AFM tip to the surface (4). The surface potential was measured in the PeakForce Kelvin mode (Kelvin probe force microscopy and its application), with a voltage applied between the two electrodes by a battery. The samples were fixed with conductive adhesive tape to the sample holder. A catalytic contact was prepared by impregnation with a solution of Pt-catalyst particles. Measurements at humidity higher than the ambient were performed in a gas tight chamber. A. Peltier element below the sample was used for heating. 


\section{Results and Discussion}

\section{Membrane electrode assemblies.}

In Figure 1, a cross section across a whole MEA with $45 \mu \mathrm{m}$ image side length measured at operating conditions of $80 \% \mathrm{RH}$ and $75{ }^{\circ} \mathrm{C}$ is presented. Electrodes, membrane and reinforcement are clearly distinguishable by their different adhesion forces. At the very left border of the sample, the embedding Teromix material is visible. In the adhesion mapping (Fig. 1b), the electrodes appear darker with a lower average adhesion. A clear contrast between the components is also observable in the stiffness mapping (Fig. 1c) with higher stiffness of the electrodes. At $50 \mathrm{mV}$ only an electronic current at the two electrodes was recorded (Fig. 1d). Already at this low resolution images, heterogeneities in the electrodes are visible.

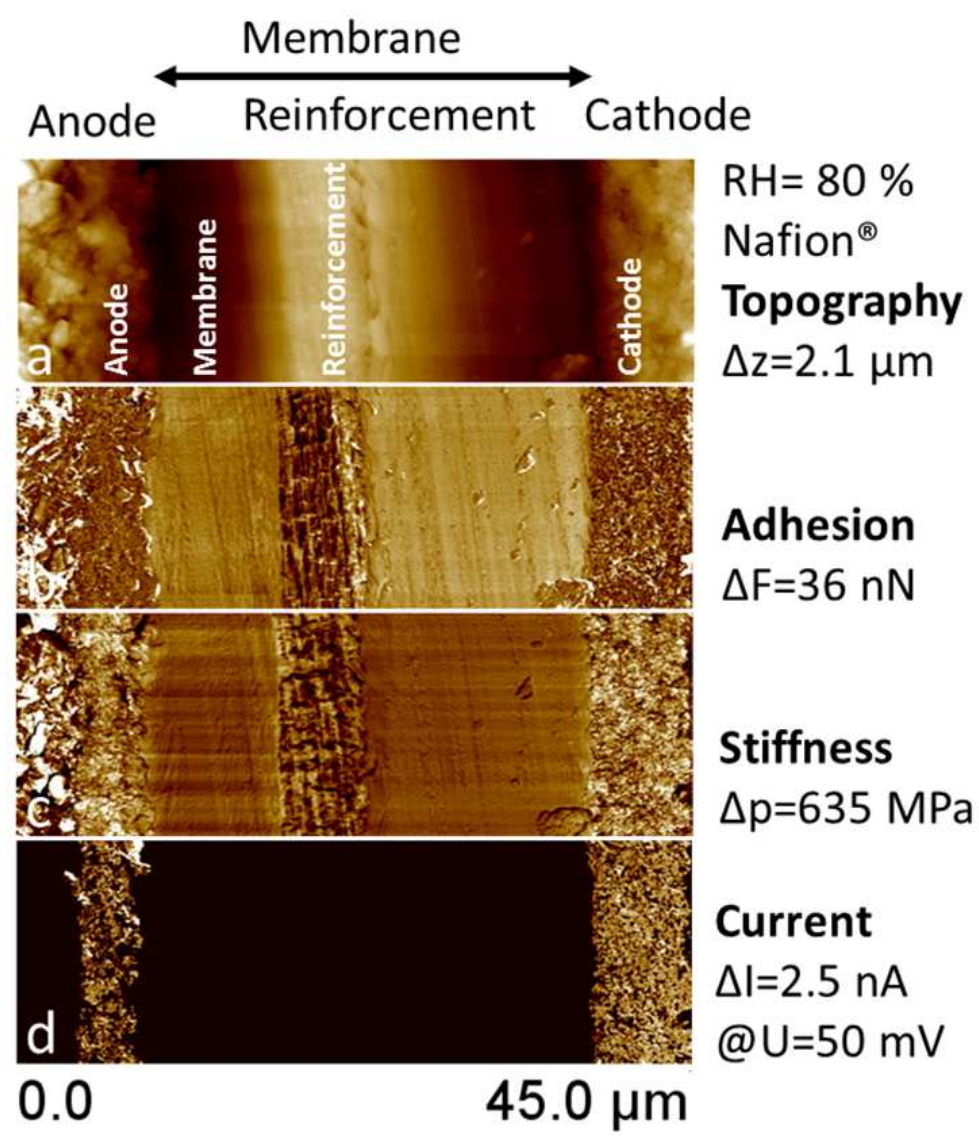

Figure 1. AFM images of a cross section of a MEA with a reinforced Nafion membrane, equilibrated at $80 \% \mathrm{RH}$, measured at $80 \% \mathrm{RH}$ and $75^{\circ} \mathrm{C}$, (a) topography of the cross section, (b) corresponding adhesion force mapping, (c) stiffness mapping, and (d) current averaged during contact of tip with surface.

At application of $2 \mathrm{~V}$ and measured with catalytically active electrodes in humid environment, in addition to the electronic current in the electrodes the ionic current was measured, shown in Figure 2. The MEA sample was operated in a fuel cell test stand for $200 \mathrm{~h}$ at $50 \% \mathrm{RH}$. The AFM measurement was performed at ambient temperature and $50 \% \mathrm{RH}$. The ionic current $(80 \mathrm{pA})$ is much lower than the electronic current that is in the 
limitations of the amplifier at the chosen amplification. At these conditions, large areas of the membrane surface are not conductive. Also in the reinforcement the conductivity is extremely low. Large, non-conductive areas have always been observed by AFM at membranes $(6,7)$.

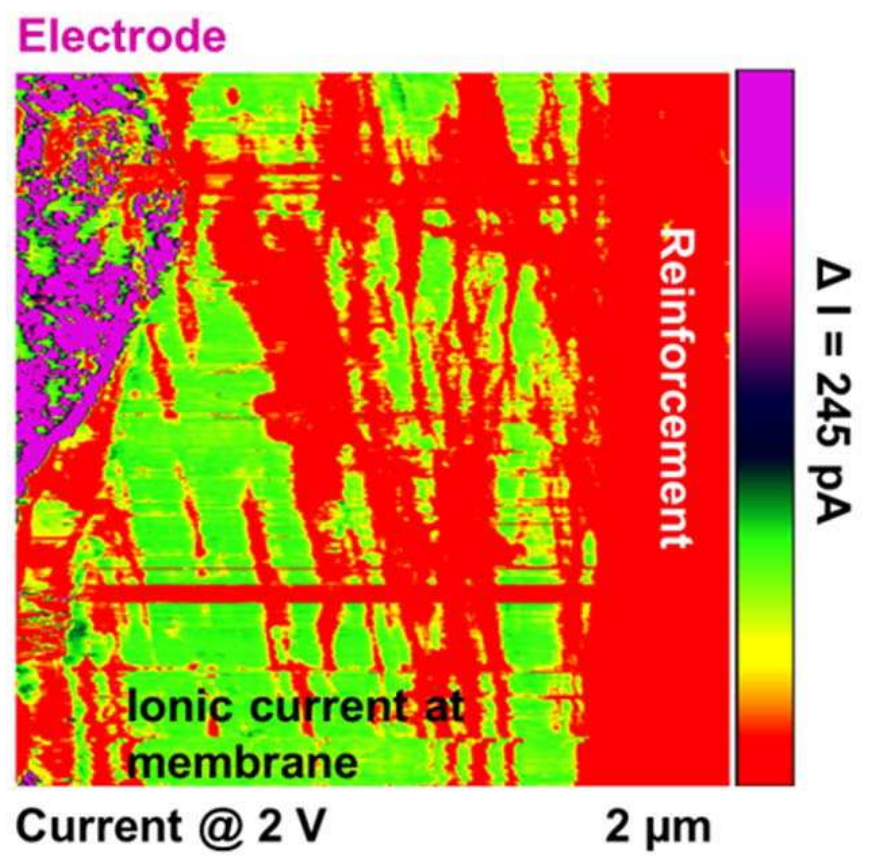

Figure 2. AFM images of a cross section of a operated MEA with a reinforced Aquivion membrane, equilibrated at $50 \% \mathrm{RH}$, measured at $50 \% \mathrm{RH}$ and room temperature, $\mathrm{U}=2$ $\mathrm{V}$, averaged during contact of tip with surface.

\section{MEA surface potential.}

In Figure 3, the surface potential across a MEA after fuel cell operation is shown as 3Dmapping. A voltage had been applied between two insulated electrodes by a battery. Due to operation conditions, platinum had been re-deposited inside the membrane in front of the cathode, analyzed by SEM. The re-deposition of platinum, mostly originating from the cathode is a well-known degradation mechanism and besides TEM and SEM measurements (8) has also been reported by AFM (9). A steep potential drop between the membrane and the electrodes is visible. The potential across the membrane stays constant at most of the surface. For two different positions; the distribution of potential across the MEA at two distinct lines is overlaid onto the complete potential image. The upper line crosses a platinum-rich area at the center of the membrane. At this position, the surface potential drops significantly. The bottom line shows the membrane potential at a position where no signs of platinum were measured. In Figure 3b, the adhesion force distribution at top of the topography image of another MEA after operation are given. The surface potential distribution is overlaid on the topography image. In contrast to Figure $3 \mathrm{a}$, the potential stays constant from the cathode up to the center of the membrane before it drops to the anode potential. As visible in the adhesion force mapping above, the surface close to the cathode was covered by a high amount of platinum particles, identified by their shape, low adhesion and high stiffness values (not shown here). This may indicate an internal electronic conductivity through the high amount of metallic particles inside the 
membrane. In this case, the electrode can be considered as effectively be shifted into the membrane and the thickness of the membrane would be effectively reduced.
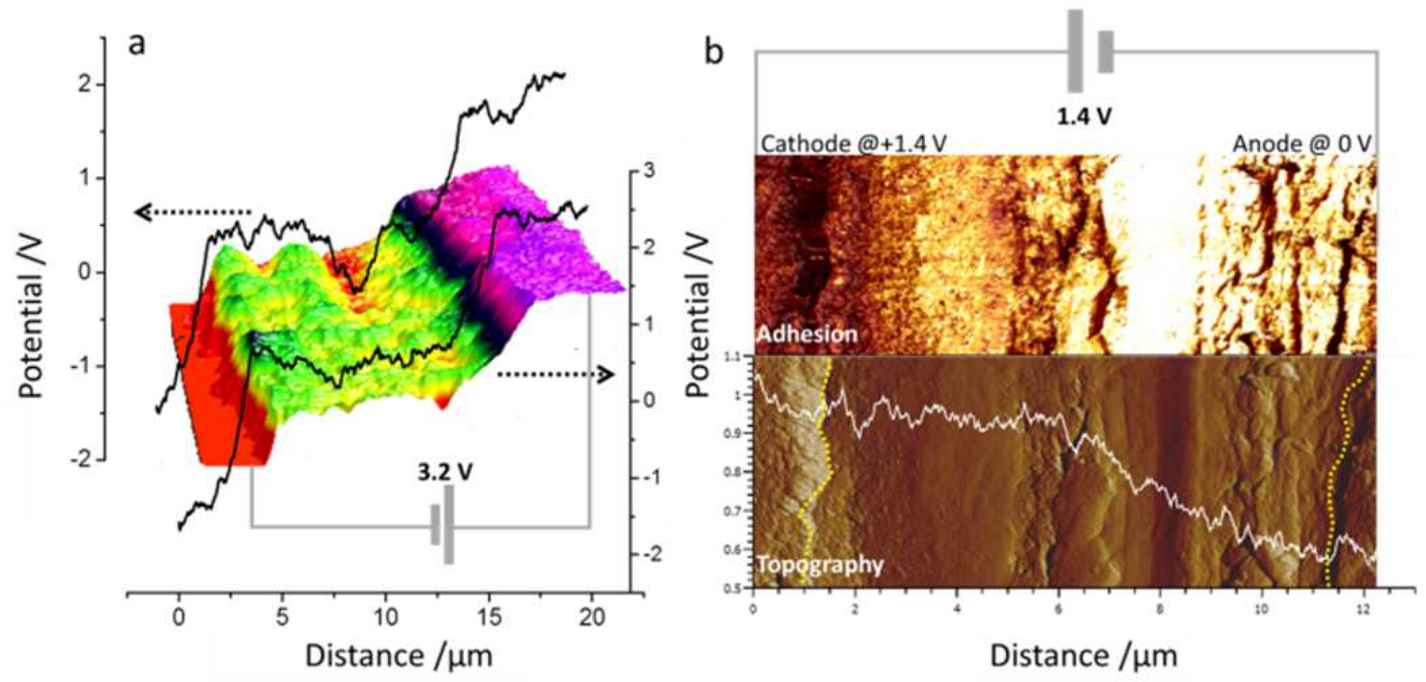

Figure 3. AFM images of a cross section of a MEA with a reinforced Nafion membrane after operation for $300 \mathrm{~h}$ at $50 \% \mathrm{RH}$ and $25^{\circ} \mathrm{C}$, topography of the cross section and overlaid surface potential with applied voltage of $1.4 \mathrm{~V}$ between the two electrodes, measured under argon/hydrogen (1\%).

\section{Electrodes - conductivity}

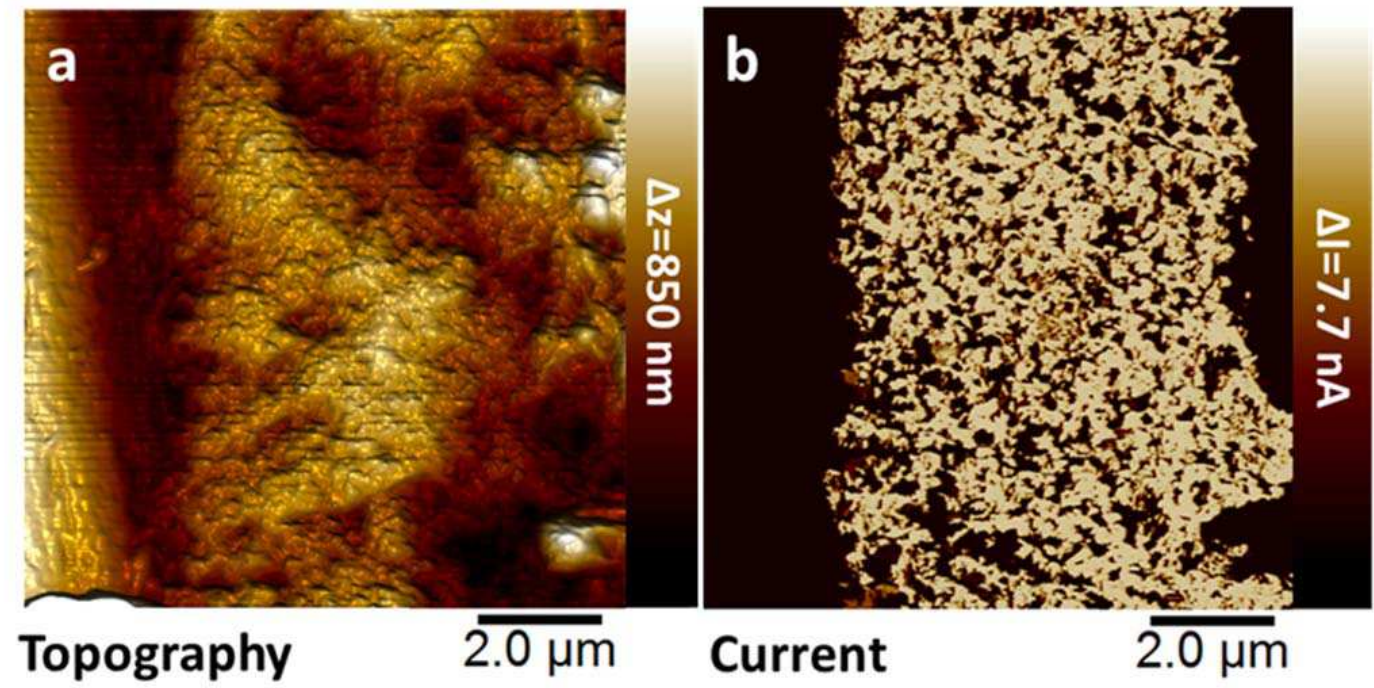

Figure 4. AFM images of a cross section of the cathode of a MEA with a reinforced Nafion membrane, equilibrated at $80 \% \mathrm{RH}$, measured at $80 \% \mathrm{RH}$ and $75^{\circ} \mathrm{C}$, (a) topography of the cross section across the cathode, (b) corresponding current image averaged during contact of tip with surface. 
In Figure 4, a higher resolution image of the cathode shown in Figure 3 with $10 \mu \mathrm{m}$ side length is given, also measured at the same operating conditions. In the current image in Figure $4 \mathrm{~b}$, large, non-conductive areas inside the electrode are visible. The reason for the non-conductive areas is either large ionomer filled part or large pores. In order to obtain more insight into this issue the conductive structure of anode and cathode are compared within the same AFM measurement; two pieces of MEA were imaged together as sketched in Figure 5a. From the current image (Figure 5b), a higher heterogeneity of the current at the anode side is already visible by the eye. An evaluation of the conductive area from the pixel values gives $69 \%$ of conductive area for the anode and $90 \%$ for the cathode side. This result is in agreement with a typically higher loading of the cathode side. The area of the yellow marked frame has been zoomed-in and the corresponding current (Fig 5c), the adhesion force (Fig 5d), and the stiffness (Fig. 5e) of this area allow a direct comparison. The non-conductive areas in the current image have a high adhesion and a low stiffness. Due to these properties they are attributed to the ionomer in the electrode.
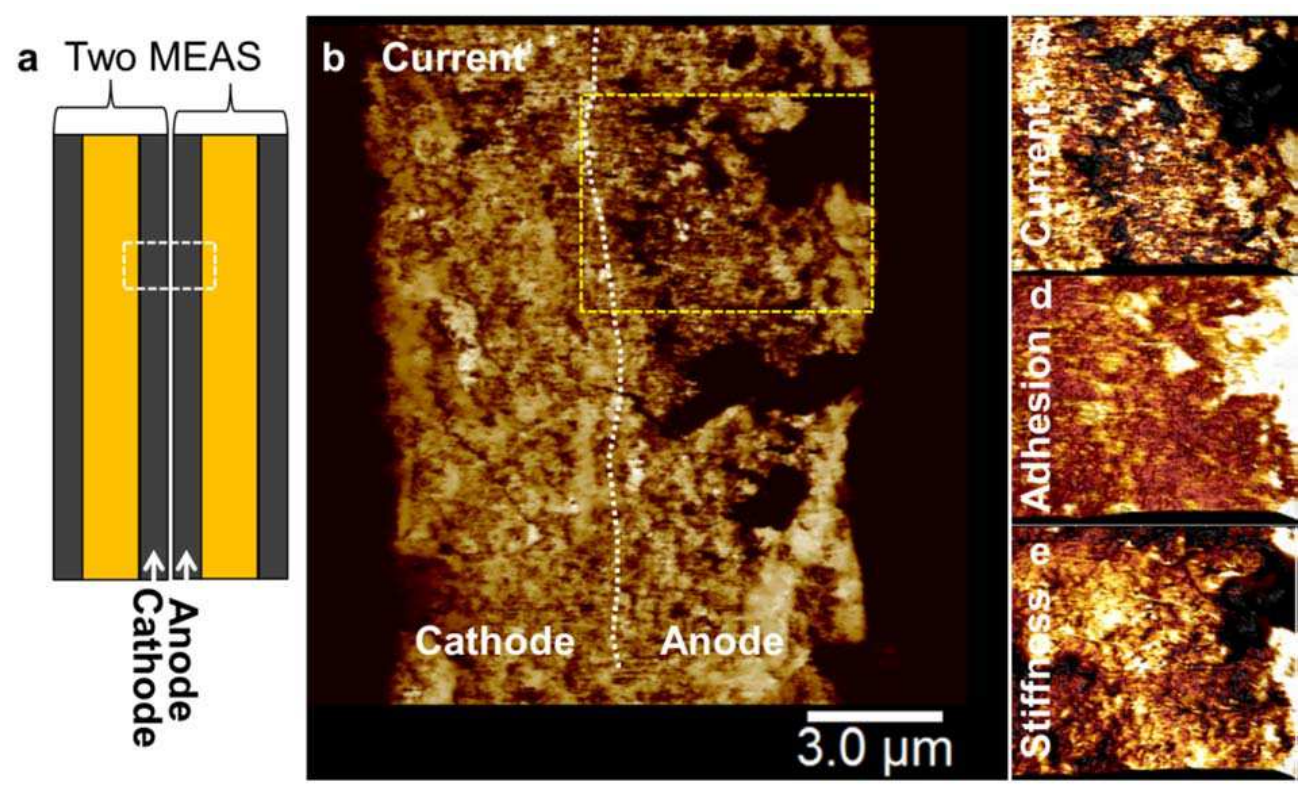

Figure 5. AFM images of a cross section across the anode and cathode of two embedded MEAs prepared with Nafion, measured at $30 \% \mathrm{RH}$ and $25^{\circ} \mathrm{C}$, (a) sketch of the preparation from two MEAs, (b) current image across cathode and anode, averaged during contact of tip with surface, (c) current of the zoomed-in area marked in yellow in (b), (d) corresponding adhesion force mapping to (c), and (e) corresponding stiffness (DMT modulus) mapping of the corresponding area of (c) and (d).

A further evaluation of the components in the electrode has been performed using current-voltage curves. $\mathrm{i}(\mathrm{U})$ curves were recorded by ramping the sample voltage at a fixed sample position. Depending on the positon, three different types of $i(U)$ curves with different resistances were distinguished, shown in figure $6 \mathrm{~b}$. They were performed at the positions indicated in Figure 6a. The lowest resistance in the range of $100 \mathrm{k} \Omega$ was attributed to the free catalyst surface that is visible as bright spots in Figure 6a, where current values were added as bright spots to the adhesion mapping and overlaid onto the 
3D-topography image. The high resistance values for the metallic contact resulted from the high resistance of the conductive adhesive tape used as fixation.
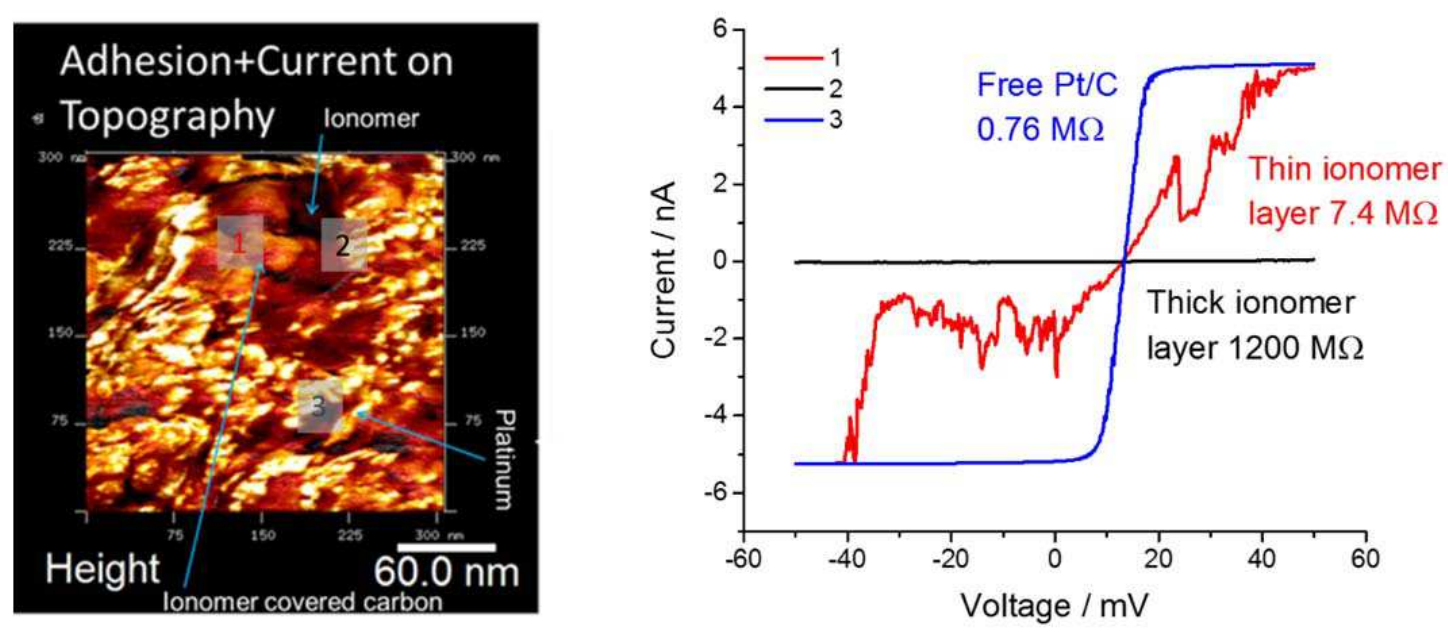

Figure 6. AFM measurement of a cross section of an electrode prepared with Aquivion ionomer, measured at $40 \% \mathrm{RH}$ and $25^{\circ} \mathrm{C}$, (a) 3D-topography image with the current values added as bright spots to the adhesion mapping overlaid onto 3D-topography, and (b) $\mathrm{i}(\mathrm{U})$ curves recorded at different positions at the electrode.

\section{Electrodes - ionomer}

The distribution of ionomer in the electrodes has been further evaluated. In Figure 7, 3Dtopography and corresponding adhesion force and stiffness mappings, measured at the cross section of an Aquivion-based electrode, are shown. As has been shown above in figures 1 and 5 , the ionomer is identified by its high adhesion force whereas $\mathrm{Pt} / \mathrm{C}$ components have a lower adhesion. Big ionomer particles that appear bright are embedded in the electrode. Numerous long fibers with lower adhesion run through the image (Fig 7b), with a more or less preferred orientation. The stiffness of the fibers (Fig. $7 \mathrm{c}$ ) does not differ from the neighboring ionomer particles (compare encircled areas in Fig. $7 \mathrm{~b}$ and c). These fibers are not typically found at electrodes. They have also been proven to be non-conductive and therefore consist of ionomer. Similar fiber-like structures have also been observed by TEM (10). Their lower adhesion force indicates a crystalline nature where sulfonic acid groups are completely turned inside to the bulk without interaction with the AFM tip. It is known that the crystalline content increases by uniaxial stretching of the ionomer (11). Therefore, it is likely that these fibers have been formed during the electrode preparation process that involved a stretching of the polymer. In combination of adhesion, stiffness and conductivity the ionomer can be unambiguously identified. 


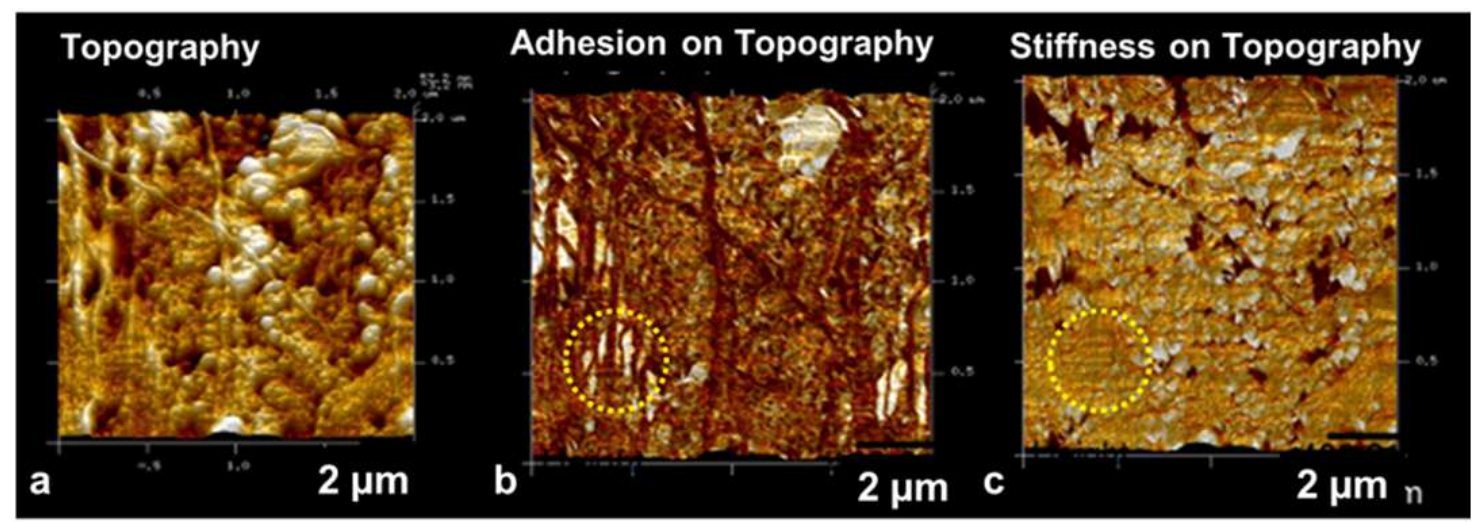

Figure 7. AFM measurement of a cross section of an electrode prepared with Aquivion ionomer, measured at $40 \% \mathrm{RH}$ and $25^{\circ} \mathrm{C}$, (a) 3D-topography image, and (b) corresponding 3D-topography image with the adhesion force values overlaid.

The ionomer content of the electrodes at the surface of the cross section has also been evaluated as shown in figure 8. In the adhesion force image (Fig 8a) the different components are visible, namely $20-30 \mathrm{~nm}$ sized mesoporous carbon particles, partly covered by platinum, identified by black color, and highly-adhesive ionomer film with bright color. Single platinum particles cannot be resolved in this image. The ionomer at the surface was evaluated by its adhesion force value. The blue-marked area with $28 \%$ of the surface in Figure $8 \mathrm{~b}$ is attributed to ionomer, measured at $25 \% \mathrm{RH}$.

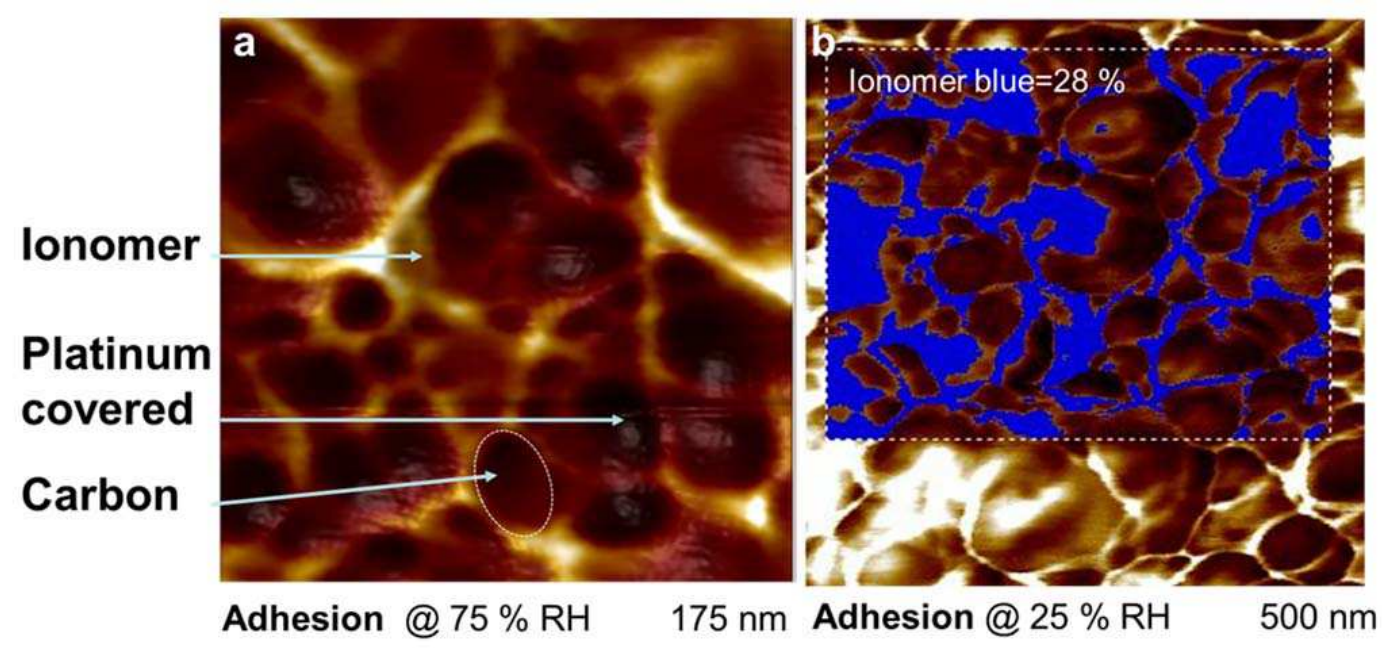

Figure 8. AFM adhesion force images of cross sections across Nafion-based electrodes, (a) measured at $75 \% \mathrm{RH}$ and $25^{\circ} \mathrm{C}$, and (b) measured at $25 \% \mathrm{RH}$, the blue marked area has been evaluated as ionomer with an area of $28 \%$ of within the frame.

From the adhesion force images, measured with a tip of $1 \mathrm{~nm}$ nominal radius and a pixel size smaller than $1 \mathrm{~nm}$, the size of the ionomer layer between the particles was measured. At $30 \% \mathrm{RH}$ and $25^{\circ} \mathrm{C}$, the smallest layer without height differences in the topography images was always determined to a range of $4-5 \mathrm{~nm}$, based on a number of different 
measurements. These sizes are in agreement with simulations reported by Borges (12) who calculated the ionomer thickness in dependence on substrate surface energy. It has been reported from the preparation of ultra-thin Nafion layers that under ambient conditions the smallest layer thickness that self-assembled from dispersion was $4 \mathrm{~nm}$ (13).

\section{Adhesion}

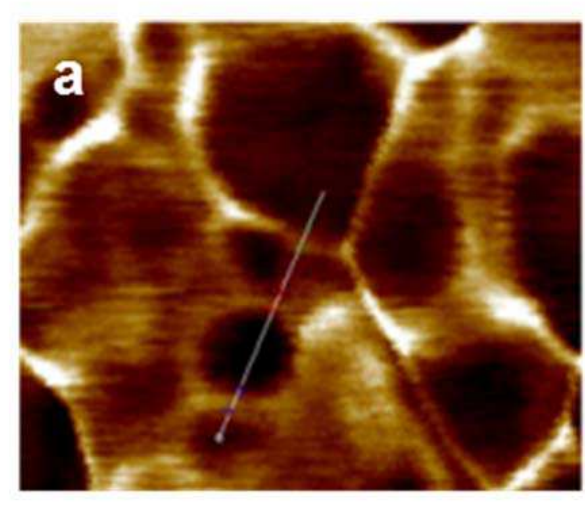

\section{$4.7 \mathrm{~nm}$}

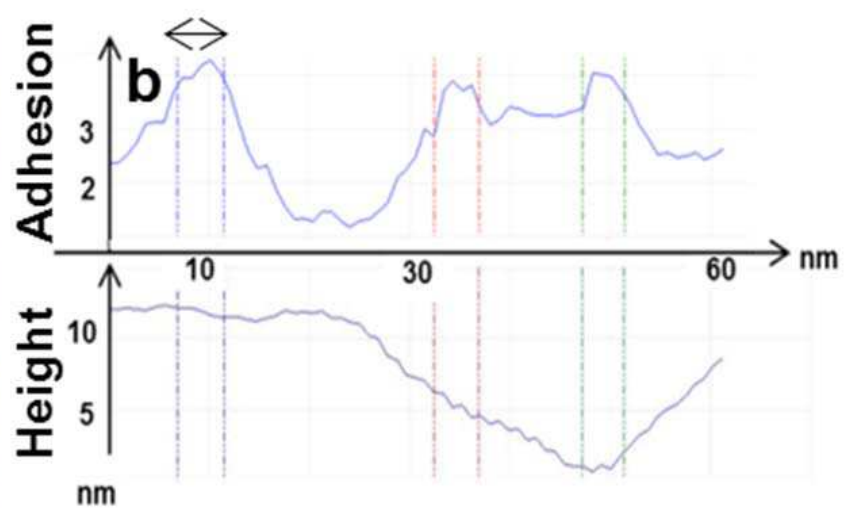

Figure 9. AFM image of a cross section of a Nafion-based electrode, measured at $40 \%$ $\mathrm{RH}$ and $25^{\circ} \mathrm{C}$, (a) adhesion force image, and (b) profile lines of adhesion force and corresponding height values along the line, marked in (a).

\section{Conclusions}

With material-sensitive and conductive AFM, the different components in MEAs can be distiguished by their properties. In principle, on the measured scale, no difference was observed between Aquivion- and Nafion-based MEAs. Carbon and Pt-covered carbon particles can be clearly distinguished from ionomer from their mechanical properties. For the first time, the investigation of a whole cross section of a fuel cell MEA and the distribution of current has been measured at fuel cell operating conditions of $80 \% \mathrm{Rh}$ and $75^{\circ} \mathrm{C}$. With AFM at cross sections that represent the electronic conductivity distribution acrosss an intact electrode, also a large heterogeneiety and larger non-conductive areas within the electrode were observed. The conductive structure and the amount of conductive area differed for anode and cathode, with $69 \%$ and $90 \%$ respectively. In agreement with electron microcopic measurements, large ionomer particles were found at cross section of electrodes. Also long fibers of ionomer with lower adhesion force than the other ionomer areas were found. Their lower adhesion force was attributed to a higher degree of crystallinity. These fibers are not always present and may result from the electrode preparation process. From the adhesion images, an evaluation of the ionomer layer thickness between $\mathrm{Pt} / \mathrm{C}$ particles was performed. At ambient condition, it resulted in a smallest layer thicknens of 4-5 nm, in agreemnet with calulations and the reported thicknes of smallest ultra-thin Nafion layers. In conclusion, the properties, escpecially concerning the water content, of the ionomer in the electrode should be different from those of a bulk membrane (14). By surface potential measurements across MEAs after 
operation, a severe influence of the re-deposited platinum on the potential distribution was found.

\section{Acknowledgments}

The research leading to these results has received funding from the European Union's Seventh Framework Programm (FP7/ 2007-2013) for the Fuel Cells and Hydrogen Joint Technology Initiative under grant agreement $\mathrm{n}^{\circ} 303452$, "IMPACTImproved Lifetime of Automotive Application Fuel Cells with ultra-low Pt-loading", and grant agreement n 621237, “ INSIDE- In -situ Diagnostics in Water Electrolyzers“

\section{References}

1. D. A. Cullen et al., J. Electrochem. Soc., 161, F1111-F1117 (2014).

2. S. Holdcroft, Chem. Mater., 26, 381-393 (2014).

3. K. More, R. Borup, and K. Reeves, in, vol. 3, p. 717-733, ECS (2006) http://ecst.ecsdl.org/cgi/doi/10.1149/1.2356192.

4. M. Lopez-Haro et al., Nat. Commun., 5, 5229 (2014).

5. R. Hiesgen et al., Membranes, 2, 783-803 (2012).

6. R. Hiesgen et al., Electrochimica Acta, 110, 292-305 (2013).

7. R. Hiesgen et al., Electrochimica Acta (2013) http://www.sciencedirect.com/science/article/pii/S0013468613008542.

8. A. Ohma, S. Yamamoto, and K. Shinohara, J. Power Sources, 182, 39-47 (2008).

9. S. Helmly et al., J. Electrochem. Soc., 160, F687-F697 (2013).

10. J. C. Idrobo et al., (2011) http://www.researchgate.net/profile/Kelly_Perry3/publication/278727328_Characteri zation_of_Fuel_Cell_Materials/links/55845ba108aeb0cdaddbbcff.pdf.

11. K. A. Mauritz and R. B. Moore, Chem. Rev., 104, 4535-4586 (2004).

12. D. Damasceno Borges, A. A. Franco, K. Malek, G. Gebel, and S. Mossa, ACS Nano, 7, 6767-6773 (2013).

13. D. K. Paul, K. Karan, A. Docoslis, J. B. Giorgi, and J. Pearce, Macromolecules, 46, 3461-3475 (2013).

14. A. Kusoglu and A. Z. Weber, in Polymers for Energy Storage and Delivery: Polyelectrolytes for Batteries and Fuel Cells, K. A. Page, C. L. Soles, and J. Runt, Editors, vol. 1096, p. 175-199, American Chemical Society, Washington, DC (2012) http://pubs.acs.org/doi/abs/10.1021/bk-2012-1096.ch011. 